\title{
Perfusion coefficient estimation from a 2D bio-heat model
}

\section{Fermín S. V. Bazán ${ }^{1}$}

Departamento de Matemática, Centro de Ciências Físicas e Matemáticas,

UFSC, Florianópolis, SC

\section{Luciano Bedin}

Departamento de Matemática, Centro de Ciências Físicas e Matemáticas,

UFSC, Florianópolis, SC

\begin{abstract}
We investigate the problem of estimating the perfusion coefficient in a $2 \mathrm{D}$ bioheat model with convective boundary conditions. As a result, a method is proposed based on a pseudoespectral method for the direct problem and the regularized Gauss-Newton for the inverse problem. Numerical results are presented.
\end{abstract}

Palavras-chave. Bioheat model, Pennes equation, regularization.

\section{Introduction}

Modeling of heat transfer processes in biological tissues is of great importance in therapeutic procedures. Accurate temperature quantification in these processes is a very difficult task due to several factors peculiar to living tissues, e.g., complex anatomical structure, blood perfusion, etc [5]. In the context of $2 \mathrm{D}$ continuum models, the temperature $U$ and the space-dependent blood perfusion coefficient $P_{f}>0$ are related through the Pennes bioheat transfer equation, [9], which in non-dimensional form, is given by

$$
\begin{aligned}
& \left.\left.U_{t}-\Delta U+P_{f} U=G, \quad(x, y) \in \mathcal{O}, t \in\right] 0, T\right], \\
& \left.\left.U_{x}=0 \quad \text { on } \quad \Gamma_{2} \times\right] 0, T\right], \\
& \left.\left.\left.U_{x}=0 \quad \text { on } \quad \Gamma_{4} \times\right] 0,\right]\right), \\
& \left.\left.U_{y}=B\left(U-U_{\infty}\right) \text { on } \quad \Gamma_{3} \times\right] 0, T\right], \\
& \left.\left.U=0 \quad \text { on } \Gamma_{1} \times\right] 0, T\right], \\
& U=T_{0} \quad \text { in } \quad \mathcal{O} \times\{0\},
\end{aligned}
$$

where $G(x, y, t)$ is a source term which stands for metabolic heat generation, $B$ is the Biot number, $U_{\infty}$ is an appropriate constant, and $T_{0}$ denotes the initial temperature. In addition, $\mathcal{O}=] 0,1[\times] 0, M\left[, \Gamma_{1}=\right] 0,1\left[\times\{M\}, \Gamma_{3}=\right] 0,1\left[\times\{0\}, \Gamma_{2}=\{0\} \times\right] 0, M[$, and $\left.\Gamma_{4}=\{1\} \times\right] 0, M[$. In general, the tissue is not homogeneous and the perfusion coefficient

\footnotetext{
${ }^{1}$ fermin.bazan@ufsc.br
} 
$P_{f}$ is time and spacewise-dependent. Several analytical and numerical methods have been employed to investigate the solution of the bioheat equation in different scenarios. For example, [4] deals with transient coefficients by spectral element methods; oscillatory heat flux condition with has been considered in [13]; in [2] the finite difference method was used in the case of concentric spherical regions, see also [12]. A 2D bioheat equation with convective boundary conditions is developed in [1].

In the inverse perfusion coefficient estimation problem, we are given a set of temperature measurements and the perfusion coefficient is regarded as unknown and needs to be estimated. Regarding this, interesting approaches for the one dimensional case can be found in [15]; an approach for the the 2D case was developed by [7]. In addition, several experimental techniques for estimating $P_{f}$ can be found in [3].

In this work we address the space-dependent perfusion estimation problem associated to the bioheat model (1)-(6), for the case where $P_{f}$ does not depend on time. The solution of this non linear inverse problem requires iterative methods where the bioheat problem (the direct problem) has to be solved at each iteration with the coefficient $P_{f}$ being considered known. In order to solve the direct problem, a pseudospectral approach is introduced and numerically illustrated in section 2. The perfusion estimation problem is outlined in Section 3. The paper ends with some considerations in section 4 .

\section{Direct Problem}

In this section we briefly describe an approach for the bioheat problem based on the pseudospectral collocation (CPS) method. The CPS approach has become an efficient way to construct approximate solutions to time dependent partial differential equations (PDEs) $[6,14]$, due to its high precision and relatively lower computation cost compared with difference finite methods. In this case, spatial derivatives are approximated by using the differentiation Chebyshev matrix, giving rise to a system of ordinary differential equations (ODEs) where only the time derivative appears, which is then integrated in time by using methods for ODEs. For simplicity we consider a mesh consisting of $(n+1) \times(n+1)$ grid points (numbered in the lexicographic ordering) based on $(n+1)$ Chebyshev-Gauss Lobatto points in each direction:

$$
x_{i}=\frac{1}{2}\left(1-\cos \frac{\pi i}{n}\right) \quad 0 \leq i \leq n, y_{j}=\frac{1}{2}\left(1-\cos \frac{\pi j}{n}\right), \quad 0 \leq j \leq n,
$$

In order to approximate spatial derivatives, let us denote the $(n+1) \times(n+1)$ differentiation Chebyshev matrix in $[0,1]$ by $D$ and assume that $D=\left[\begin{array}{llll}d_{0} & d_{1} & \cdots & d_{n}\end{array}\right]=$ $\left[\begin{array}{lll}r_{0} & r_{1} \cdots r_{n}\end{array}\right]^{T}, d_{i}, r_{i} \in \mathbb{R}^{n+1}, D_{1}=\left[\begin{array}{llll}d_{1} & d_{2} & \cdots & d_{n-1}\end{array}\right]$, and $D_{2}=\left[\begin{array}{lll}r_{1} & r_{2} \cdots r_{n-1}\end{array}\right]^{T}$. Let $\bar{U}_{j}(t)=\left[U\left(x_{0}, y_{j}, t\right), \quad U\left(x_{1}, y_{j}, t\right), \cdots U\left(x_{n}, y_{j}, t\right)\right]^{T}, \quad 0 \leq j \leq n$. Then the vector second 
order derivatives of $U$ with respect to $x$ can be approximated as

$$
\left[\begin{array}{c}
U_{x x}\left(x_{0}, y_{0}, t\right) \\
\vdots \\
U_{x x}\left(x_{n}, y_{0}, t\right) \\
\vdots \\
U_{x x}\left(x_{0}, y_{n-1}, t\right) \\
\vdots \\
U_{x x}\left(x_{n}, y_{n-1}, t\right)
\end{array}\right] \approx\left(I_{n} \otimes D_{1} D_{2}\right) \bar{U}(t)
$$

where $\bar{U}(t)=\left[\bar{U}_{0}(t)^{T} \bar{U}_{1}(t)^{T} \cdots \bar{U}_{n-1}(t)^{T}\right]^{T}$, and where $\otimes$ stands for Kronecker product. Similarly, the vector containing second order derivatives with respect to $y$ in all points of the grid can be approximated by

$$
\left[\begin{array}{c}
U_{y y}\left(x_{0}, y_{0}, t\right) \\
\vdots \\
U_{y y}\left(x_{n}, y_{0}, t\right) \\
\vdots \\
U_{y y}\left(x_{0}, y_{n-1}, t\right) \\
\vdots \\
U_{y y}\left(x_{n}, y_{n-1}, t\right)
\end{array}\right] \approx\left[\left(B \bar{d}_{0} e_{1}^{T}+\bar{D}_{1} \bar{D}_{2}\right) \otimes I_{(n+1)}\right] \bar{U}(t)-B U_{\infty} \bar{H}
$$

where $\bar{H}=\left[H_{0}^{T}, \ldots, H_{n-1}^{T}\right]^{T}$, with $H_{i}=e_{i+1}^{T} \bar{d}_{0}[1, \ldots, 1]^{T} \in \mathbb{R}^{n+1}, \quad i=0, \ldots, n-1$. In the above equation $\bar{D}_{1}$ and $\bar{D}_{2}$ are defined similarly as $D_{1}$ and $D_{2}$, respectively, where $\bar{d}_{i}$ (resp. $\bar{r}_{i}$ ) is obtained by taking the first $n$ components of $d_{i}$ (resp. $r_{i}$ ). Neglecting discretization errors and denoting the vector of approximations to $\bar{U}(t)$ by $V(t)$, (8), (9) and (1) yield an initial-value problem of the form

$$
\left\{\begin{array}{l}
V^{\prime}(t)=A V(t)+S(t) \\
V(0)=U_{0}
\end{array}\right.
$$

where

$$
\begin{gathered}
A=\left[\left(I_{n} \otimes D_{1} D_{2}\right)+\left(B \bar{d}_{0} e_{1}^{T}+\bar{D}_{1} \bar{D}_{2}\right) \otimes I_{(n+1)}-\bar{P}_{f}\right] \\
\bar{P}_{f}=\operatorname{diag}\left(P_{f}\left(x_{0}, y_{0}\right), \ldots, P_{f}\left(x_{n}, y_{0}\right), \ldots, P_{f}\left(x_{0}, y_{n-1}\right), \ldots, P_{f}\left(x_{n}, y_{n-1}\right)\right)
\end{gathered}
$$

and

$$
\begin{gathered}
S(t)=\bar{F}(t)-B U_{\infty} \bar{H}, \quad \text { with } \\
\bar{F}(t)=\left[F\left(x_{0}, y_{0}, t\right), \ldots, F\left(x_{n}, y_{0}, t\right), \ldots, F\left(x_{0}, y_{n-1}, t\right), \ldots, F\left(x_{n}, y_{n-1}, t\right)\right]^{T} .
\end{gathered}
$$

Thus, application of the CPS-based numerical approach to the bio-heat model reduces to apply time integration methods for ODES for solving the initial value problem (10). In our computations we integrate in time by the fourth order Runge-Kutta method which we denote by CPS-RK4. To illustrate the effectiveness of the CPS-RK4 approach we 
consider the perfusion coefficient constant case where the source term is taken to be

$$
\begin{aligned}
G(x, y, t) & =e^{a t} y^{2}(y-M) \cos (\pi x)[a \cos (c t)-c \sin (c t)] \\
& -e^{a t} \cos (c t) \cos (\pi x)\left[-\pi^{2} y^{2}(y-M)+(6 y-2 M)\right]-\frac{2 B U_{\infty}}{M} \\
& +P_{f}\left[e^{a t} \cos (c t) y^{2}(y-M) \cos (\pi x)+\frac{B U_{\infty}}{M} y(y-M)\right]
\end{aligned}
$$

where $a, c$ are arbitrary real constants. In this case the solution for this bioheat problem can be shown to be $U(x, y, t)=e^{a t} \cos (c t) y^{2}(y-M) \cos (\pi x)+\frac{B U_{\infty}}{M} y(y-M)$. We report results corresponding to the data $a=-50, c=3 \pi, B=0.015, P_{f}=0.1, \quad M=1$, and $U_{\infty}=0.001$. Discretization is made taking $n=20$, which implies a grid of $21 \times 21$ points and a system matrix $A$ of size $420 \times 420$. We compute the maximum allowable timestep for stable integration and use $\Delta t=0.00004$. The results are displayed in Figure 1 .
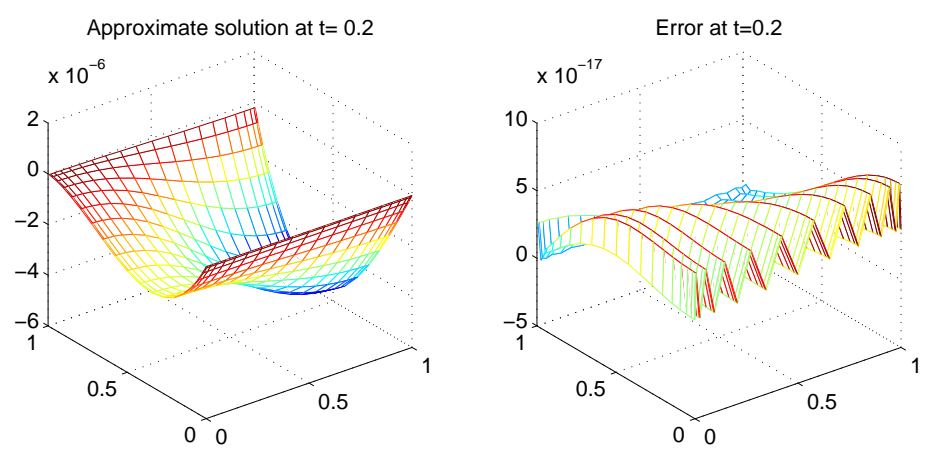

Figure 1: Approximate solution computed by CPS-RK4 and corresponding error with respect to the exact solution in a grid of $21 \times 21$ points.

\section{Perfusion coefficient estimation: pseudospectral approach}

In practice the goal is to estimate the perfusion coefficient by using a set of measured temperature as input data. The estimation approach proposed in this paper assumes that the initial-boundary value problem (1)-(6) is transformed into a system of ordinary differential equations (ODEs) in which only the time derivative appears. This means that discretization is made only in space and that, due to the nature of the original problem, the discretization procedure gives rise to a system of ODEs of the form

$$
\left\{\begin{array}{l}
\mathrm{U}^{\prime}(t)=\mathrm{A}(p) \mathrm{U}(t)+\mathrm{S}(t) \\
\mathrm{U}(0)=U_{0}
\end{array}\right.
$$

where $p$ is a vector of unknown parameters, $A(p)$ is a square matrix that depends non linearly on $p$ and the chosen discretization method, and the source term $S(t)$ is a vector valued function that also depends on the chosen spatial discretization method. The solution of (10) is a vector valued function that depends on $\mathbf{p}$ and contains approximations to 
the solution $U\left(t, x_{i}, y_{j}\right)$ on the spatial grid; this solution is denoted by $U(p, t)$. Thus, the inverse problem of estimating the perfusion coefficient can be formulated as one of estimating a vector $p$ of parameters such that the difference between computed temperatures $U(p, t)$ at prescribed locations and experimentally acquired temperatures at the same locations is minimized in some sense. For future reference, computed and experimentally measured temperatures at locations $\ell_{i}, i=1,2, \ldots, M$, and time levels $t_{k}, k=1, \ldots, q$, are denoted by $U_{\ell_{i}}^{t_{k}}(p)$ and $\widetilde{U}_{\ell_{i}}^{t_{k}}$, respectively.

Thus the perfusion estimation problem can be, in principle, handled by solving a non linear least squares problem of the form

$$
p^{*}=\underset{p \in \mathbb{R}^{N}}{\operatorname{argmin}} F(p), \quad F(p)=\sum_{k=1}^{q} \sum_{i=1}^{M}\left(U_{\ell_{i}}^{t_{k}}(p)-\widetilde{U}_{\ell_{i}}^{t_{k}}\right)^{2},
$$

where $p=\left[p_{1}, \ldots, p_{N}\right]^{T}$ is the vector of unknowns. Differentiation of $F$ with respect to $p_{j}$ gives

$$
\frac{\partial F(p)}{\partial p_{j}}=2 \sum_{k=1}^{q} \sum_{\ell=1}^{M}\left(\widetilde{U}_{\ell_{i}}^{t_{k}}(p)-\widetilde{U}_{\ell_{i}}^{t_{k}}\right) \frac{\partial U_{\ell_{i}}^{t_{k}}(p)}{\partial p_{j}} .
$$

Hence the necessary condition of minimum, $\frac{\partial F(p)}{\partial p_{j}}=0, j=1,2, \ldots, N$, can be rewritten in matrix form as

$$
2 J(p)^{T}(U(p)-\widetilde{U})=0,
$$

where $J(p)$ is an $(M \times q) \times N$ referred to as the sensitivity matrix and given as

$$
J(p)=\left(\begin{array}{c}
J_{1}(p) \\
\vdots \\
J_{q}(p)
\end{array}\right), \quad \text { with } \quad\left[J_{k}(p)\right]_{i, j}=\frac{\partial U_{\ell_{i}}^{t_{k}}(p)}{\partial p_{j}}, 1 \leq i \leq M, 1 \leq j \leq N .
$$

The non linear problem (16) must be solved iteratively with the matrix $J(p)$ changing at each iteration. Therefore, the matrix $J(p)$ must be computed efficiently and this can be done as follows. Taking partial derivative with respect to $p_{j}$ on both sides of (10) gives

$$
\frac{\partial}{\partial p_{j}} U^{\prime}(t)=A(p) \frac{\partial U(p, t)}{\partial p_{j}}+\frac{\partial A(p)}{\partial p_{j}} U(p, t)
$$

Letting $V(p)(t)=\partial U(p, t) / \partial p_{j}$ and interchanging the order of differentiation, the above equation becomes an system of ODEs with $V(t)$ as unknown. On the other hand, if (10) is solved for $U(t)$ then it follows that $\partial U(p, t) / \partial p_{j_{\mid}=0}=0$. Hence, the determination of the $j$ th column of the sensitivity matrix requires solving the initial value problem

$$
\left\{\begin{array}{l}
V^{\prime}(t)=A(p) V(t)+W(t) \\
V(0)=0
\end{array}\right.
$$

where $W(t)=\partial A(p) / \partial p_{j} U(p, t)$. Once this initial value problem is solved, the $j$ th column of the sensitivity matrix is determined by taking the components of $V(t)$ that correspond 
to the locations $l_{i}, i=1, \ldots, M$. Notice that this requires solving the direct problem (10) for $U(p, t)$. As already mentioned, in this paper we assume that the direct problem (1)-(6) is solved by using the pseudospectral collocation method, as described in the previous section, and thus, for each $p$, this gives rise to a system matrix given by

$$
A(p)=\left[\left(I_{n} \otimes D_{1} D_{2}\right)+\left(B \bar{d}_{0} e_{1}^{T}+\bar{D}_{1} \bar{D}_{2}\right) \otimes I_{(n+1)}-\bar{P}_{f}\right],
$$

where $\bar{P}_{f}$ is a diagonal matrix given as

$$
\bar{P}_{f}=\operatorname{diag}\left(P_{f}\left(x_{0}, y_{0}\right), \ldots, P_{f}\left(x_{n}, y_{0}\right), \ldots, P_{f}\left(x_{0}, y_{n-1}\right), \ldots, P_{f}\left(x_{n}, y_{n-1}\right)\right),
$$

thereby indicating that the vector of parameters is

$$
p=\left[P_{f}\left(x_{0}, y_{0}\right), \ldots, P_{f}\left(x_{n}, y_{0}\right), \ldots, P_{f}\left(x_{0}, y_{n-1}\right), \ldots, P_{f}\left(x_{n}, y_{n-1}\right)\right]^{T} .
$$

Having computed efficiently the sensitivity matrix, the nonlinear problem (16) can be handled in several ways. For example by using non linear conjugate gradients (NCG), Gauss-Newton methods, Tikhonov regularization, or others. For an account of a variety of methods for heat inverse problems, the reader is referred to [8]. In this work, the perfusion estimation problem is addressed through a regularized Gauss-Newton method.

\section{Conclusions}

A two dimensional Pennes equation subjected to mixed boundary conditions in a rectangle has been considered focusing, first, on a numerical method for the direct problem. The numerical method transforms the bioheat problem into a first order system of ordinary differential equations where only the time derivative appears, and then integrates in time by using the fourth order Runge-Kutta method. The method was illustrated numerically and shown to be very efficient in terms of simplicity and accuracy. In addition, the perfusion estimation problem was addressed by using a regularized version of the Gauss-Newton method. The development of further methods for this inverse problem is the subject of ongoing research.

\section{Acknowledgements}

This work was supported by CNPq, Brazil, grant 307476/2014-6

\section{References}

[1] M. D. B. Azevedo, R. O. C. Guedes and F. Scofano Neto, Analytical solution to the two dimensional transient bioheat equation with convective boundary conditions, Proceedings of the 11 ENCIT, Paper CIT06-0605, (2006).

[2] H. G. Bagaria and D. T. Johnson, Transient solution to the bioheat equation and optimization for magnetic fluid hyperthermia treatment, Int. J. of Hyperthermia, vol. 21 , pp. 57-75, (2005). 
[3] A. V. Cardinali, Validation of a Noninvasive Blood Perfusion Measurement Sensor, Master Thesis in Mechanical Engineering, Faculty of the Virginia Polytechnic Institute and State University, (2002).

[4] M. Deghan and M. Sabouri, A spectral element method for solving the Pennes bioheat transfer equation by using triangular and quadrilateral elements, App. Math. Modelling, 36, pp. 6031-6049, (2012).

[5] J. Fan and L. Wang, Analytical theory of bioheat transport, J. Appl. Phys., vol. 109, pp. 1-43, (2011).

[6] B. Fornberg, A Practical Guide to Pseudospectral Methods, Cambridge University Press, Cambridge, (1996).

[7] C. F. Lopes, M. V. C. Souza, F. Scofano Neto, M. J., Colaço, A. B. Caldeira, and R. O. C. Guedes, Inverse Problem of Estimation of the Blood Perfusion Coefficient in Cancerous Tissues Subjected to Hyperthermic Treatments. EngOpt 2008- International Conference on Engineering Optimization, Rio de Janeiro, (2008).

[8] M. N. Ozisik and H. R. B. Orlande, Inverse heat transfer, Taylor \& Francis, New York, (2000).

[9] H. H. Pennes, Analysis of tissue and arterial blood temperatures in the resting human forearm, Journal of Applied Physiology, vol. 1(2), pp. 93-122, (1948).

[10] S. Reddy and L. N. Trefethen, Stability analysis of the method of lines, Numer. Math., 62 , p. 237-267, (1992).

[11] K. A. Shah and P. H. Bhathawala, Analytical solution of malignant skin tumor problem by Green's function method, Int. J. of Appl. Math and Mech., vol. 8(17), pp. 98-111, (2012)

[12] W. Shen and J. Zhang, Modeling and numerical simulation of bioheat transfer and biomechanics in soft tissue, Math. Comput. Model., vol. 41, pp. 1251-1265, (2005).

[13] T. C., Shih. Y. Ping, W. Lin, W. and H. Kou, Analytical analysis of the Pennes bioheat transfer equation with sinusoidal heat flux condition on skin surface, Medical Engineering \& Physics, vol. 29, pp. 946-953, (2007).

[14] L. N. Trefethen, Spectral Methods in Matlab, SIAM, Philadelphia, PA, (2000).

[15] D. Trucu, D. B. Ingham, ans D. Lesnic, Space-dependent perfusion coefficient identification in the transient bio-heat equation, Journal of Eng. Math. (67) pp. 307-315, (2010). 